\title{
Genetic Diversity and Phyletic Evolution of Eleven Chinese Indigenous and Three Commercial Chicken Breeds by mtDNA Sequences
}

\section{-Author(s)}

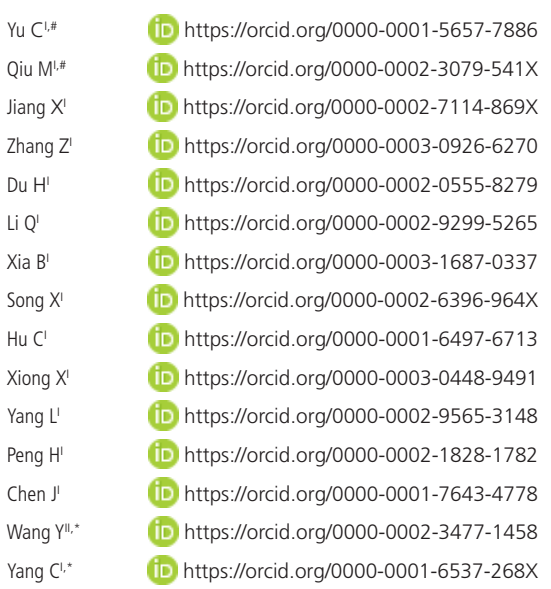

Animal Breeding and Genetics Key Laboratory of Sichuan Province, Sichuan Animal Science Academy, 7\# Niusha Road, Chengdu 610066 China

" Farm Animal Genetic Resources Exploration and Innovation Key Laboratory of Sichuan Province, Sichuan Agricultural University, 211 Huiming road, Chengdu 611130, China.

\#These authors contributed equally to this work. *Corresponding author

\section{Mail Address}

Corresponding author e-mail address Chaowu Yang

Animal Breeding and Genetics Key Laboratory of Sichuan Province, Sichuan Animal Science Academy, 7\# Niusha Road, Chengdu 610066, China.

Phone: 008602884555593

Email: cwyang@foxmail.com

\section{neywords}

Chinese chicken, Mitochondrial DNA, Cluster analysis, Gene flow, Phylogeny.

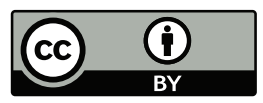

Submitted: 23/April/2019 Approved: 27/August/2019

\section{ABSTRACT}

Chinese indigenous chicken breeds are geographically widespread, and a total of 116 indigenous chicken breeds are listed as Chinese national genetic resources. However, these indigenous chicken breeds are facing serious challenges as declining population and germplasm degeneration because lots of commercial chicken breeds had been introduced. In this study, the genetic variations of eleven Chinese indigenous chicken breeds of Sichuan province and three commercial chicken breeds were investigated based on the partial mitochondrial DNA D-loop of 487bp in length. 147 individuals from 14 breeds were examined and 34 haplotypes were observed. Genetic diversity analysis showed that the highest haplotype diversity level was found in Dahen Chicken (DH) population, while the Arbor Acres Chicken (WF) and Roman layer (RM) showed lower genetic diversity levels. The long-term artificial selection may lead to reduced nucleotide diversity. Genetic population differentiation analysis indicated that most of the variation $(80.80 \%)$ was attributed to variations among breeds. Phylogenetic analysis revealed that these individuals were divided into four distinct genetic clades, including cluster A, B, C and D. Eighteen haplotypes were classified as cluster $A$, eight haplotypes were classified as cluster $B$, five haplotypes were classified as cluster $C$ and three haplotypes were classified as cluster D. There was no breed-specific clade. Our study firstly identified the population's genetic structure of Chinese indigenous chickens and the most important commercial breeds in Sichuan province, though the genetic diversity of indigenous breeds did not suffer obvious decrease, but could be helpful for efficient artificial breeding selection and genetic resources conservation.

\section{INTRODUCTION}

Due to the wide area and the diversity of the natural ecological conditions in China, different chicken breeds have emerged. A total of 116 indigenous chicken breeds have been listed as Chinese national genetic resources. These indigenous chickens are usually preferred by Chinese customers due to their taste, flavor, leanness and good disease resistance (Izadi et al., 2011; Okumu et al., 2017). One obvious characteristic of the indigenous chicken in China is their extensive phenotypic variation in colors (plumage, earlobe and comb), comb types, morphology and body size. Sichuan province is an important development area in China, with mild climate and abundant resources. Besides, lots of chicken breeds have evolved with local characteristics. Some breeds have beautiful appearance, some produce green-shell eggs, and some have good meat flavor. In a word, these indigenous chicken breeds have a high economic value to marginal farmers. For example, Sichuan Mountain Black-bone Chicken and Muchuan Black-bone Chicken have a high nutritional value, and Pengxian 
Yu C, Qiu M, Jiang X, Zhang Z, Du H, Li $Q$, Xia B, Song $X$, Hu C, Xiong $X$, Yang $L$, Peng $H$, Chen J, Wang $Y$, Yang $C$

\section{Genetic Diversity and Phyletic Evolution of Eleven Chinese Indigenous and Three Commercial Chicken Breeds by mtDNA Sequences}

Yellow Chicken has good adaptability traits as well as juicy meat characteristics. However, the rapid economic development and improved transportation in the southwest have left indigenous chickens in disadvantage compared with the commercial chickens with fast growth rates and high egg production. As a result, many indigenous chicken breeds are either extinct or seriously replaced by globally used highly productive breeds. The decline in the genetic diversity of chicken population has become a serious problem. Therefore, it is important to study the genetic diversity of both indigenous and commercial chicken breeds (Wu et al., 2014).

Mitochondrial DNA (mtDNA) and microsatellites are two types of molecular information that have been widely used to evaluate the genetic diversity of various animal species (Groeneveld et al., 2010). Due to the mitochondrial D-loop region with the largest variation and highest mutation rate of mtDNA, mitochondrial DNA has been regarded as an appropriate marker to study the genetic diversity and was widely used to analyze the population genetics in ducks (Li et al., 2010), Egyptian indigenous chicken (Eltanay and Hemeda, 2016), Mediterranean chicken (Ceccobelli et al., 2015) and other animals (Czerneková et al., 2013; Ivanković et al., 2014). Animal mtDNA is inherited maternally and evolves 5-10 times faster than nuclear genetic markers (Brown et al., 1982), because it appears in multiple copies in the cells and the mitochondrial gene content is strongly conserved across generations (Di Lorenzo et al., 2015). Therefore, mtDNA has become a useful marker for the study of genetic variability and population structure in livestock and animal pathogens (Muchadeyi et al., 2008; Walker et al., 2007). Previous studies of chicken mtDNA have revealed that Mediterranean chickens originated from the Red Jungle Fowl type (Gallus gallus) (Moiseyeva et al., 2003). Liu et al. (2006a) found that there were 169 haplotypes through the study of the mitochondrial DNA hypervariable segment I (HVS-I) of domestic chickens across Eurasia as well as wild red jungle fowls from Southeast Asia and China. Mwacharo et al. (2011) found 5 haplogroups in East Africa chickens, of which $D$ haplogroup has the largest proportion. In addition, Ceccobelli et al. (2015) has also used mtDNA and microsatellites to investigate the evolutionary relationship of 16 chickens breeds from the northern shores of the Mediterranean. However, few systematic studies about the genetic diversity of indigenous chicken in Sichuan province (China) have been reported.
Therefore, the objectives of the present study were to assess the genetic diversity, population structure and the phylogenic relationship of eleven economically important indigenous chicken breeds and three commercial chicken breeds using the mtDNA sequence. The result can be used in formulating national plans and strategies for sustainable improvement of these important chicken breeds in Sichuan province. In addition, it might also provide a genetic tool for chicken breeding and offer a scientific support for resources conservation.

\section{MATERIALS AND METHODS}

\section{Sample collection and DNA extraction}

Blood samples of 147 chickens from 11 indigenous and 3commercial chicken breeds, including Sichuan Mountain Black-bone Chicken (SD), Muchuan Blackbone Chicken (MC), Jiuyuan Black Chicken (JY), Emei Black Chicken (EM), Tibetan Chicken ( $T$ ), Luning Chicken (LN), Miyi Chicken (MY), Jingyang Silky Chicken (JY), Yanying Chicken (YY), Pengxian Yellow Chicken (PXY), Caoke Chicken (CK), Arbor Acres Chicken (WF), Rohman layer(RM) and Dahen Chicken (DH) were used as DNA source in this study. Among these chicken breeds, WF and RM are the foreign commercial breeds. $\mathrm{DH}$ is a native commercial breed bred by Sichuan Dahen Poultry Breeding Co., Ltd. Located in Hanchang Town, Chengdu City of Sichuan Province. The detailed information of the sample is listed in Table 1. All blood samples were taken from the wing vein $(2 \mathrm{ml}$ for each animal, collected with Vacutainer tubes containing EDTA as anticoagulant) and stored at $-20^{\circ} \mathrm{C}$ prior to DNA extraction. DNA was extracted from whole blood using the Axygen blood kit (Corning, USA) and stored at $-20^{\circ} \mathrm{C}$ until sequencing. In addition, 34 published Sichuan indigenous chicken sequences were included in the study (samples information and the haplotypes are listed in Table 1).

\section{Mitochondrial DNA D-loop amplification and sequencing}

For the phylogenetic analysis, we
amplified the mitochondrial hyper-variable segment I using forward primer L16750:5'AGGACTACGGCTTGAAAAGC-3' and reverse primer H522:5' -ATGTGCCTGACCGAGGAACCAG-3' (Fu et al., 2001; Liu et al., 2006a). L and $\mathrm{H}$ refer to light and heavy strands, respectively. The reaction of PCR amplification was performed using a Verity 96well (Applied $A B I$, USA) in a final volume of $50 \mu$ l with $1 \mu$ l of 
Table 1 - Sample information and the haplotypes(147 birds)of 14 chicken breeds.

\begin{tabular}{|c|c|c|c|c|}
\hline ID & Breeds & Haplotypes & No. & Location \\
\hline 1 & CK & $\mathrm{H} 1(11), \mathrm{H} 4(1), \mathrm{H} 9(2), \mathrm{H} 10(1), \mathrm{H} 12(1), \mathrm{H} 15(2), \mathrm{H} 25(1), \mathrm{H} 32(2)$ & 21 & Ya'an,Sichuan, China \\
\hline 2 & SD & $\begin{array}{l}\mathrm{H} 1(2), H 5(1), H 10(1), H 15(4), H 16(1), H 18(1), H 26(1), H 27(3), H 29(1), \\
H 30(1), H 32(5)\end{array}$ & 21 & Yibin, Sichuan, China \\
\hline 3 & MC & $\mathrm{H} 15(1), \mathrm{H} 23(1), \mathrm{H} 32(4)$ & 6 & Leshan, Sichuan, China \\
\hline 4 & JY & $\mathrm{H} 2(1), \mathrm{H} 11(1), \mathrm{H} 17(2), \mathrm{H} 25(1), \mathrm{H} 32(3), \mathrm{H} 33(1)$, & 9 & Dazhou, Sichuan, China \\
\hline 5 & EM & $\mathrm{H} 1(4), \mathrm{H} 3(1), \mathrm{H} 6(1), \mathrm{H} 8(1), \mathrm{H} 31(1), \mathrm{H} 32(3)$ & 11 & Leshan, Sichuan, China \\
\hline 6 & T & $\mathrm{H} 11(4), \mathrm{H} 17(1), \mathrm{H} 2 \mathrm{O}(1), \mathrm{H} 28(1), \mathrm{H} 31(2), \mathrm{H} 32(3), \mathrm{H} 33(2)$ & 14 & Ganzi Tibetan autonomous prefecture, Sichuan, China \\
\hline 7 & LN & $\mathrm{H} 19(2), \mathrm{H} 21(1), \mathrm{H} 22(1), \mathrm{H} 23(2), \mathrm{H} 32(4)$ & 10 & Liangshan Yi Autonomous Prefecture, Sichuan, China \\
\hline 8 & MY & $\mathrm{H} 1(1), \mathrm{H} 15(2), \mathrm{H} 17(1), \mathrm{H} 24(1), \mathrm{H} 32(5)$ & 10 & Panzhihua, Sichuan, China \\
\hline 9 & JYS & $\mathrm{H} 7(1), \mathrm{H} 12(1), \mathrm{H} 13(1), \mathrm{H} 23(4), \mathrm{H} 32(1), \mathrm{H} 34(1)$ & 9 & Liangshan Yi Autonomous Prefecture, Sichuan, China \\
\hline 10 & YY & $\mathrm{H} 1(3), \mathrm{H} 12(1), \mathrm{H} 14(1), \mathrm{H} 23(2), \mathrm{H} 25(1)$ & 8 & Liangshan Yi Autonomous Prefecture, Sichuan, China \\
\hline 11 & PXY & $\mathrm{H} 1(3), \mathrm{H} 31(1)$ & 4 & Chengdu, Sichuan, China \\
\hline 12 & WF & $\mathrm{H} 15(7), \mathrm{H} 17(2), \mathrm{H} 31(1)$ & 10 & Chengdu, Sichuan, China \\
\hline 13 & RM & $\mathrm{H} 31(3), \mathrm{H} 33(5)$ & 8 & Chengdu, Sichuan, China \\
\hline 14 & $\mathrm{DH}$ & $\mathrm{H} 1(1), \mathrm{H} 5(1), \mathrm{H} 15(1), \mathrm{H} 31(1), \mathrm{H} 32(2)$ & 6 & Chengdu, Sichuan, China \\
\hline
\end{tabular}

Note: $C K=$ Caoke Chicken; SD=Sichuan Mountain Black-bone Chicken;MC=Muchuan Black-bone Chicken; JY=Jiuyuan Black Chicken; EM=Emei Black Chicken;T=Tibetan Chicken, $\mathrm{LN}=$ Luning Chicken; $\mathrm{MY}=$ Miyi Chicken;JYS=Jingyang Silky Chicken; $Y Y=$ Yanying Chicken; $\mathrm{PXY}=$ Pengxian Yellow Chicken; WF=Arbor Acres Chicken; $R M=R o m a n$ layer; $D H=D a h e n$ Chicken; $\mathrm{H1}$ =haplotype 1, H2=haplotype 2,.....H34=haplotype 34; Accession No. of H1to H28 in Genbank is MN365166 to MN365193; Accession No. of H29 to H34 is AF512261,AF512262, AF512263, AF512091, AY465960, AY465961 separately.

each primer $(10 \mathrm{pmol} / \mu \mathrm{l}), 1 \mu \mathrm{l}$ of DNA template $(100 \mathrm{ng} /$ $\mu \mathrm{l}), 1 \mu \mathrm{dNTP}(10 \mathrm{mM}$ each)0.5 $\mu \mathrm{l}$ TaqDNA Polymerase (5 U/ul) (Sangon Biotech Co., Ltd, Shanghai, China), $5 \mu \mathrm{l}$ 10xPCR Buffer, $5 \mu \mathrm{l} \mathrm{MgCl}_{2}(25 \mathrm{mM})$ and 35.5 $\mu \mathrm{l} \mathrm{ddH}_{2} \mathrm{O}$. The amplification reaction was done in 35 cycles, each one including denaturation at $94^{\circ} \mathrm{C}$ for 30s, annealing at $59^{\circ} \mathrm{C}$ for $30 \mathrm{~s}$ and extension at $72^{\circ} \mathrm{C}$ for $30 \mathrm{~s}$. Furthermore, we also performed a step of denaturation at $95^{\circ} \mathrm{C}$ for 3 min and a stage of extension at $72^{\circ} \mathrm{C}$ for $10 \mathrm{~min}$. The purity of DNA fragments was checked by agarose gel electrophoresis, and the DNA concentration was measured with a spectrophotometer. The PCR products were purified with a DNA purification kit (Sangon Biotech Co., Ltd, Shanghai, China), following the manufacturer's instructions and sent to Sangon Biotechnology Co., Ltd (Shanghai, China) for sequencing. The obtained sequence data have been submitted to Genbank (Accession number: MN365166-193).

\section{Data analysis}

The raw sequences obtained were edited and aligned using the DNAstar package (DNASTAR), all sequences were aligned with the $G$. gallus sequence available in GenBank (X52392) using the ClustalW method included in the MEGA7.0 program (Kumar et al., 2016). MEGA 7.0 program was used to estimate the sequence comparisons and variation analysis. The haplotype sequences were used for subsequent analyses. The composition of nucleotides, the number of haplotypes (h), haplotype diversity $(H)$, nucleotide diversity (Pi), average number of nucleotide differences (k) and average number of nucleotide substitutions (Dxy) per site were estimated using DNASP 5.10 (Librado and Rozas, 2009). The phylogenetic trees of the haplotypes were constructed with the neighbor-joining (NJ) method implemented in MEGA 7.0. Robustness of phylogenetic hypotheses was calculated based on 1000 replications (Felsenstein, 1985). And the Meleagris gallopavo mtDNA D-loop sequence (AY398680) was used as the outgroup in the phylogenetic tree. In addition, the median-joining (MJ) network of the haplotypes was constructed and plotted using Network 5.0 (Bandelt et al., 1999).

Genetic variation among and within breeds was estimated with an analysis of molecular variance (AMOVA) in Arlequin 3.5.12 (Excoffier et al., 2005). The fixation indices $\left(F_{\mathrm{ST}}\right)$ and significance between breeds were also calculated in Arlequin 3.5.12. The calculation formula of gene flow between breeds was $\mathrm{Nm}=\left(1 / \Phi_{\mathrm{ST}}-1\right) / 2$. Meanwhile, the neutrality tests such as Tajima's D and Fu's Fs statistic were also calculated using Arlequin 3.5.12. The mismatch analysis was implemented in DNASP 5.10 to find whether the breeds underwent a population expansion.

\section{RESULTS}

\section{mtDNA D-loop sequence variability and nucleotide composition}

We amplified and sequenced the 588-bp fragments, of which the 484-bp sequences of HSV-I were used in subsequent analyses (from nt 16 to nt 499 of X52392 sequence). In 147 indigenous chickens, the average 
Yu C, Qiu M, Jiang X, Zhang Z, Du H, Li Q, Xia B, Song $X$, Hu C, Xiong X, Yang L, Peng H, Chen J, Wang Y, Yang C

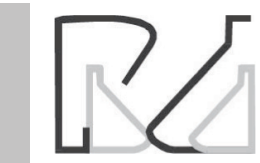

Genetic Diversity and Phyletic Evolution of Eleven Chinese Indigenous and Three Commercial Chicken Breeds by mtDNA Sequences nucleotide composition was $27.7 \%$ A, 13.2\% G, 30.3\% $\mathrm{C}$ and $28.8 \% \mathrm{~T}$, contents $\mathrm{A}+\mathrm{T}$ (56.5\%) was higher than that of $C+G(43.5 \%)$, showing that $A$ and $T$ were richer in the chicken mtDNA-loop region. There were 77 polymorphic sites with 27 singleton polymorphic sites and 50 parsimony-informative polymorphic sites. The variable types were transitions, transversions, and 3-bpinsertion was detected at the $52^{\text {nd }}$ bases with CCC in our sequences. Totally, 34 haplotypes were found in 14chicken breeds, as shown in Table 2.

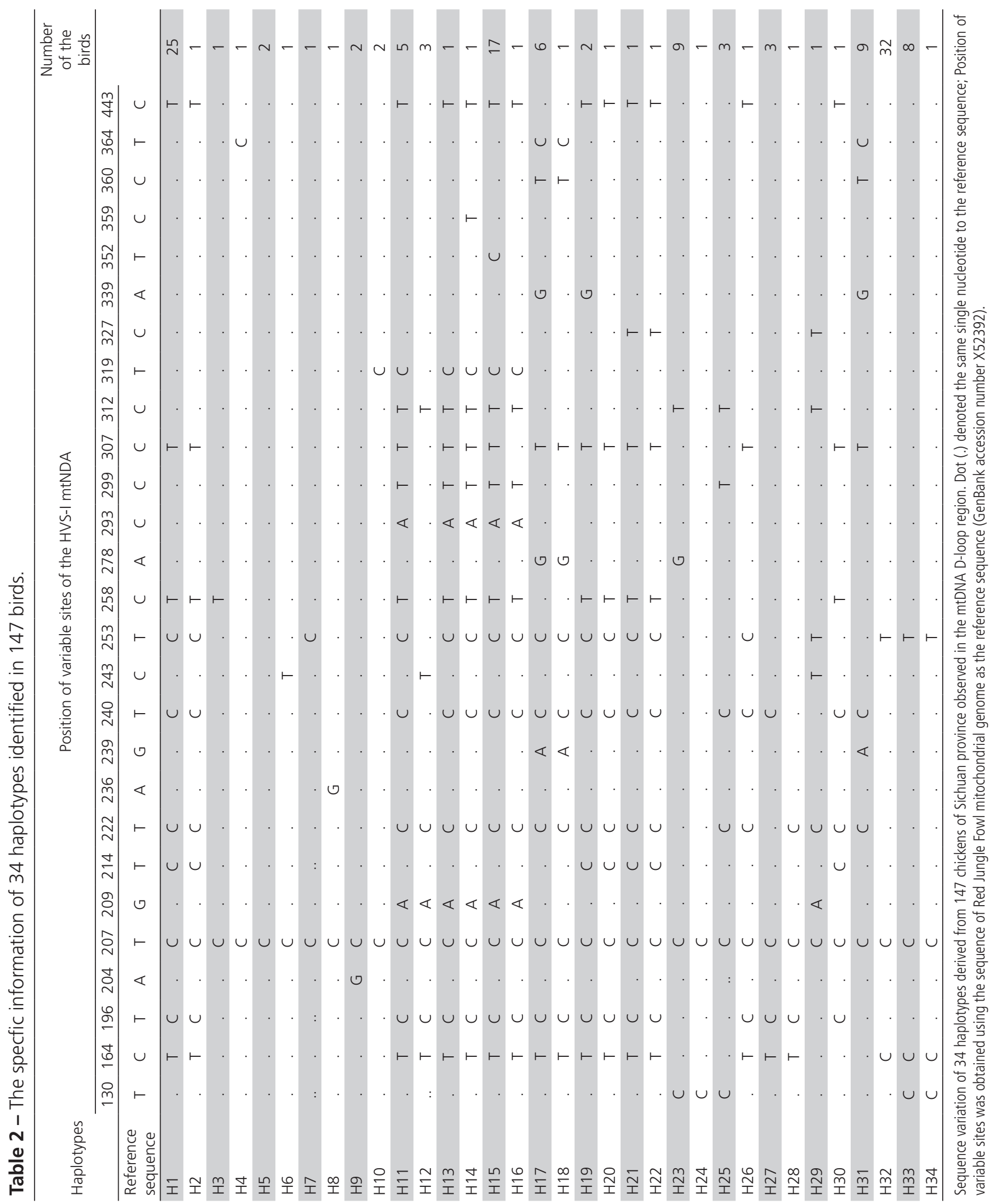


The genetic diversity of 14 breeds was shown in Table 3. Among 34 haplotypes, 22 were unique haplotypes detected in the 147 individuals, and other haplotypes were shared by more than two breeds. The diverged nucleotide diversity and haplotype diversity were exhibited in different chicken breeds. The nucleotide diversity $(\mathrm{Pi})$ values ranged from 0.00333 to 0.02504 and the overall value was 0.01362 for fourteen breeds. The lowest nucleotide diversity was in the RM breed and the highest one was in the $Y Y$ breed. The EM, PXY and CK breeds had the similar nucleotide diversity of $0.00996,0.00934$ and 0.00913 , respectively. Meanwhile, the value of the haplotype diversity $(\mathrm{Hd})$ ranged from 0.5000 to 0.9333 , which the lowest was in the PXY breed and the highest was in the DH breed.

\section{Nucleotide divergence and Net genetic distance among breeds}

Nucleotide divergence (Dxy) and net genetic distance ( $\mathrm{Da}$ ) were important indexes for the evaluation of nucleotide polymorphism. Within the 14 chicken breeds, the average Dxy was 0.0138 (rang from 0.0058 to 0.0244 ) and the average Da was 0.0028 (range from 0.0001 to 0.0115 ) (Table 4). The largest Da (0.0115)
Table 3 - Genetic diversity parameters estimated from 14 chicken breeds in the Sichuan province.

\begin{tabular}{lcccccc}
\hline Breed & $\mathrm{N}$ & $\mathrm{S}$ & $\mathrm{h}$ & $\mathrm{Hd}$ & $\mathrm{Pi}$ & $k$ \\
\hline SD & 21 & 21 & 11 & 0.90476 & 0.01221 & 5.88571 \\
MC & 6 & 7 & 3 & 0.60000 & 0.00484 & 2.33333 \\
JY & 9 & 20 & 6 & 0.88889 & 0.01510 & 7.27778 \\
EM & 11 & 12 & 6 & 0.83636 & 0.00996 & 4.80000 \\
T & 14 & 19 & 7 & 0.79120 & 0.01327 & 6.39560 \\
LN & 10 & 9 & 5 & 0.82222 & 0.00821 & 3.95556 \\
MY & 10 & 12 & 5 & 0.75556 & 0.00862 & 4.15556 \\
JYS & 9 & 16 & 6 & 0.83333 & 0.01544 & 7.44444 \\
YY & 8 & 36 & 5 & 0.85714 & 0.02504 & 12.08143 \\
PXY & 4 & 9 & 2 & 0.50000 & 0.00934 & 4.50000 \\
CK & 21 & 9 & 8 & 0.72381 & 0.00913 & 4.40000 \\
WF & 10 & 11 & 3 & 0.51111 & 0.00802 & 3.86667 \\
RM & 8 & 3 & 2 & 0.53571 & 0.00333 & 1.60714 \\
DH & 6 & 11 & 5 & 0.93333 & 0.01093 & 5.26667 \\
Total & 147 & 49 & 34 & 0.90029 & 0.01362 & 6.56546 \\
\hline
\end{tabular}

Note: N: Number of sequence used, S: Number of segregation site, h: Number of haplotypes, Hd: haplotype diversity, Pi: nucleotide diversity, k: average number of differences.

was found between RM and CK chicken breeds, and the smallest Da (0.0001) was observed between $T$ and SD, YY and JYS population, respectively. Meanwhile, the largest Dxy (0.0244) was found between RM and YY chicken breeds, and the smallest Dxy (0.0058) was observed between RM and MC breeds.

Table 4 - Genetic distance and nucleotide divergence between 14 different chicken breeds.

\begin{tabular}{lccccccccccccccc}
\hline$D_{x y} / D_{A}$ & $S D$ & $M C$ & $J Y$ & $E M$ & $T$ & $L N$ & $M Y$ & $J Y S$ & $Y Y$ & $P X Y$ & $C K$ & $W F$ & $R M$ & $D H$ \\
\hline$S D$ & & 0.0099 & 0.0134 & 0.0128 & 0.0128 & 0.0149 & 0.0102 & 0.0165 & 0.0213 & 0.0133 & 0.0142 & 0.0107 & 0.0109 & 0.0109 \\
MC & 0.0013 & & 0.0123 & 0.0133 & 0.0106 & 0.0082 & 0.0071 & 0.0136 & 0.0219 & 0.0149 & 0.0161 & 0.0069 & 0.0058 & 0.0086 \\
JY & -0.0003 & 0.0024 & & 0.0127 & 0.0134 & 0.0162 & 0.0116 & 0.0163 & 0.0205 & 0.0127 & 0.0136 & 0.0129 & 0.0132 & 0.0123 \\
EM & 0.0018 & 0.0059 & 0.0002 & & 0.0124 & 0.0178 & 0.0112 & 0.0155 & 0.0177 & 0.0084 & 0.0094 & 0.0134 & 0.0146 & 0.0108 \\
T & 0.0001 & 0.0016 & -0.0008 & 0.0008 & & 0.0146 & 0.0108 & 0.0154 & 0.0205 & 0.0126 & 0.0137 & 0.0119 & 0.0113 & 0.0113 \\
LN & 0.0047 & 0.0017 & 0.0046 & 0.0087 & 0.0038 & & 0.0121 & 0.0131 & 0.0231 & 0.0191 & 0.0200 & 0.0121 & 0.0117 & 0.0139 \\
MY & -0.0002 & 0.0004 & -0.0003 & 0.0019 & -0.0002 & 0.0037 & & 0.0145 & 0.0202 & 0.0119 & 0.0130 & 0.0083 & 0.0086 & 0.0089 \\
JYS & 0.0027 & 0.0035 & 0.0010 & 0.0028 & 0.0011 & 0.0013 & 0.0024 & & 0.0203 & 0.0156 & 0.0161 & 0.0157 & 0.0170 & 0.0153 \\
YY & 0.0027 & 0.0070 & 0.0004 & 0.0002 & 0.0014 & 0.0065 & 0.0034 & 0.0001 & & 0.0166 & 0.0172 & 0.0225 & 0.0244 & 0.0200 \\
PXY & 0.0025 & 0.0078 & 0.0005 & -0.0013 & 0.0013 & 0.0103 & 0.0029 & 0.0031 & -0.0006 & & 0.0081 & 0.0145 & 0.0160 & 0.0111 \\
CK & 0.0035 & 0.0091 & 0.0015 & -0.0002 & 0.0025 & 0.0114 & 0.0041 & 0.0038 & 0.0001 & -0.0012 & & 0.0153 & 0.0177 & 0.0123 \\
WF & 0.0006 & 0.0005 & 0.0014 & 0.0044 & 0.0013 & 0.0040 & -0.0001 & 0.0039 & 0.0059 & 0.0058 & 0.0068 & & 0.0086 & 0.0098 \\
\hline RM & 0.0031 & 0.0017 & 0.0040 & 0.0079 & 0.0030 & 0.0059 & 0.0026 & 0.0076 & 0.0103 & 0.0096 & 0.0115 & 0.0029 & & 0.0097 \\
DH & -0.0007 & 0.0008 & -0.0007 & 0.0003 & -0.0008 & 0.0004 & -0.0009 & 0.0021 & 0.0020 & 0.0009 & 0.0023 & 0.0003 & 0.0026 &
\end{tabular}

Note: Lower diagonal is nucleotide divergence (Da); upper diagonal was net genetic distance (Dxy).

\section{Analysis of Molecular Variance (AMOVA) and gene flow}

Molecular variance (AMOVA) based on the mtDNA D-loop sequences of 14 chicken breeds were analyzed. The results showed that the $19.20 \%$ of the genetic variation derived from variation among the breeds, while most of the variation ( $80.80 \%)$ was attributed to variation within breeds (Table 5). Fst value is an indicator of genetic differentiation between the breeds. As shown in Table 5, the Fst of $0.1920(p<0.01)$ among all the breeds implied that the genetic divergence within breeds was significant. The highest Fst value was found between RM and PXY $(0.708, p<0.05)$, followed by Fst value from RM and CK $(0.579, p<0.05)$, and the 
Table 5 - Analysis of molecular variance (AMOVA) between D-loop sequences haplotypes in Sichuan Province chicken breeds.

\begin{tabular}{lcccc}
\hline Source of variation & d.f. & Sum of squares & Variance components & Percentage of variation (\%) \\
\hline Among breeds & 13 & 119.425 & $0.6321 \mathrm{Va}$ & 19.20 \\
Within breeds & 133 & 353.717 & $2.6595 \mathrm{Vb}$ & 80.80 \\
Total & 146 & 473.143 & 3.2917 & \\
Fixation index (Fst) & & & $0.1920(\mathrm{P}=0.0000)$ & \\
\hline
\end{tabular}

lowest differentiation was found between $T$ and SD (0.006, p>0.05) (Table 6).

In addition, gene flow among the different breeds was estimated by $\mathrm{Nm}$. (Table 6). We found that consistent with the Fst analyses, Nm showed restricted gene flow between the sampled localities. In different chicken breeds, distinct gene exchange
(Fst=0.006, $\quad \mathrm{Nm}=71.666 ; \quad \mathrm{Fst}=0.006, \quad \mathrm{Nm}=82.404)$ was detected between SD and T, PXH and JY breeds, respectively. However, at the same geography region, obvious genetic differentiation was found in Liangshan prefecture breeds $(p<0.05)$ while conspicuous gene exchange (Fst=0.009, $\mathrm{Nm}=56.91)$ was only found in YY and JYS chickens.

Table 6 - Population pairwise Fst values and gene flow (Nm) between 14 chicken breeds based on D-loop sequence.

\begin{tabular}{lccccccccccccccc}
\hline Fst/Nm & SD & MC & JY & EM & $T$ & LN & MY & JYS & YY & PXY & CK & WF & RM & DH \\
\hline SD & & 6.095 & inf & 3.372 & 71.666 & 1.197 & inf & 2.356 & 2.469 & 2.707 & 1.506 & 11.872 & 3.215 & inf \\
MC & 0.076 & & 2.712 & 0.725 & 4.7865 & 2.209 & 14.154 & 1.788 & 1.344 & 0.408 & 0.468 & 9.293 & 4.349 & 5.182 \\
JY & -0.016 & 0.156 & & 27.134 & $\inf$ & 1.246 & $\inf$ & 7.694 & 21.674 & 82.404 & 3.157 & 4.083 & 1.429 & inf \\
EM & 0.129 & 0.408 & 0.018 & & 7.919 & 0.526 & 2.469 & 2.148 & 17.479 & inf & inf & 1.021 & 0.505 & 13.577 \\
\hline T & 0.006 & 0.094 & $-0.058^{*}$ & 0.059 & & 1.497 & $\inf$ & 6.406 & 5.145 & 6.805 & 2.105 & 4.449 & 2.353 & inf \\
LN & $0.295^{*}$ & 0.184 & 0.287 & 0.487 & 0.250 & & 1.136 & 4.280 & 1.213 & 0.408 & 0.389 & 1.014 & 0.590 & 1.036 \\
MY & -0.030 & 0.034 & -0.020 & $0.168^{*}$ & $-0.023^{*}$ & $0.306^{*}$ & & 2.404 & 2.268 & 1.462 & 1.093 & inf & 2.672 & inf \\
JYS & $0.175^{*}$ & $0.218^{*}$ & 0.061 & 0.189 & 0.072 & 0.105 & $0.172^{*}$ & & 56.91 & 2.601 & 1.351 & 1.445 & 0.730 & 3.548 \\
YY & $0.168^{*}$ & $0.271^{*}$ & 0.023 & 0.028 & 0.089 & 0.292 & 0.181 & $0.009^{*}$ & & inf & 8.895 & 1.308 & 0.789 & 6.158 \\
PXY & 0.156 & $0.550^{*}$ & 0.006 & -0.160 & 0.068 & 0.551 & $0.254^{*}$ & $0.161^{*}$ & -0.091 & & inf & 0.702 & 0.206 & 6.013 \\
CK & $0.249^{*}$ & $0.517^{*}$ & $0.137^{*}$ & -0.016 & $0.192^{*}$ & $0.562^{*}$ & $0.314^{*}$ & $0.270^{*}$ & 0.053 & -0.141 & & 0.647 & 0.364 & 1.990 \\
WF & 0.040 & 0.051 & 0.109 & $0.331^{*}$ & 0.101 & $0.330^{*}$ & -0.008 & $0.257^{*}$ & $0.277^{*}$ & $0.416^{*}$ & $0.436^{*}$ & & 1.840 & 11.909 \\
RM & $0.135^{*}$ & 0.103 & $0.260^{*}$ & $0.498^{*}$ & 0.175 & $0.459^{*}$ & 0.158 & $0.406^{*}$ & $0.388^{*}$ & $0.708^{*}$ & $0.579^{*}$ & $0.214^{*}$ & \\
DH & -0.068 & 0.088 & -0.063 & 0.036 & -0.077 & $0.326^{*}$ & -0.098 & 0.124 & 0.075 & 0.077 & $0.201^{*}$ & 0.040 & 0.199 & 2.014 \\
\hline
\end{tabular}

Note: Lower diagonal is nucleotide divergence (Fst); upper diagonal is net genetic distance $(\mathrm{Nm})$, inf represents the net genetic distance between the two breeds is infinite, ${ }^{*} p<0.05$ means significant difference between the two breeds.

\section{Population demography history estima- tion}

In order to trace the recent demographic events, Tajima's D and Fu's Fs neutrality tests were performed. Usually, significant positive values indicate population subdivision or population bottleneck, conversely, the significant negative values indicate population expansion or purifying selection. As shown in Table 7, Tajima's D values were not significantly different in all breeds, indicating that neither balancing selection nor purifying selection occurred in all chicken breeds. Fu's Fs values of the SD and DH breeds were -0.925 and -0.259 , respectively, but not significant from a statistic point of view.

The mismatch distribution analysis for all breeds showed a multi-peak (Figure 1), which suggests that there was no rapid expansion event that occurred in the 14 chicken's population demographic history.
In all simulation runs, there were positive and nonsignificant values of the Sum of squared deviations, SSD $(0.132, p=0.176)$ and Harpending's Raggedness index, $r(0.233, p=0.456)$.

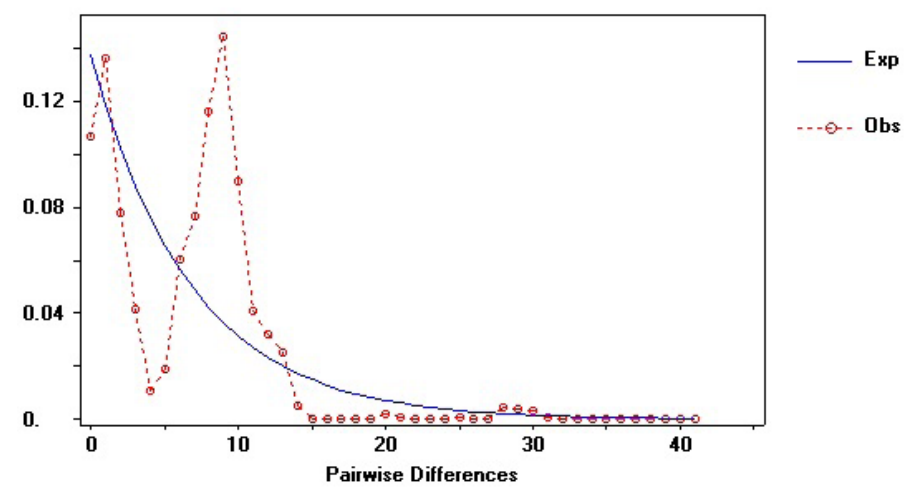

Figure 1 - Mismatch-distribution to test the expansion of 14 chicken breeds in Sichuan province. The number of nucleotide differences between pairs of sequences is indicated along the $\mathrm{X}$-axis, and their frequency along the $\mathrm{Y}$-axis. The straight line represents the expected values and the dotted line represents the observed values. 
Yu C, Qiu M, Jiang X, Zhang Z, Du H, Li $Q$, Xia B, Song $X$, Hu C, Xiong $X$, Yang $L$, Peng H, Chen J, Wang Y, Yang C

\section{Genetic Diversity and Phyletic Evolution of Eleven Chinese Indigenous and Three Commercial Chicken Breeds by mtDNA Sequences}

Table 7 - Population dynamics and expansion for Sichuan province chicken breeds.

\begin{tabular}{lcccc}
\hline Population & Tajima's D & Fu's Fs & SSD & $r$ \\
\hline SD & $0.031(0.572)$ & $-0.925(0.353)$ & $0.045(0.05)$ & $0.041(0.370)$ \\
MC & $-1.390(0.051)$ & $1.312(0.750)$ & $0.097(0.290)$ & $0.204(0.700)$ \\
JY & $-0.054(0.515)$ & $0.832(0.638)$ & $0.101(0.020)$ & $0.312(0.040)$ \\
EM & $0.745(0.797)$ & $0.620(0.632)$ & $0.114(0.080)$ & $0.146(0.330)$ \\
T & $0.293(0.654)$ & $1.222(0.715)$ & $0.115(0.060)$ & $0.066(0.360)$ \\
LN & $1.066(0.883)$ & $0.977(0.716)$ & $0.056(0.420)$ & $0.206(0.180)$ \\
MY & $-0.092(0.475)$ & $1.098(0.714)$ & $0.045(0.240)$ & $0.075(0.670)$ \\
JYS & $1.283(0.921)$ & $0.883(0.648)$ & $0.122(0.090)$ & $0.114(0.380)$ \\
YY & $-0.789(0.254)$ & $3.037(0.904)$ & $0.500(0.000)$ & $0.222(0.150)$ \\
PXY & $-0.829(0.115)$ & $3.777(0.939)$ & $0.047(0.480)$ & $0.750(0.920)$ \\
CK & $-0.624(0.290)$ & $0.486(0.636)$ & $0.412(0.000)$ & $0.078(0.480)$ \\
WF & $-0.025(0.510)$ & $3.925(0.953)$ & $0.031(0.220)$ & $0.540(0.930)$ \\
RM & $1.667(0.921)$ & $0.866(0.590)$ & $0.134(0.170)$ & $0.292(0.450)$ \\
DH & $0.564(0.713)$ & $-0.259(0.367)$ & $0.132(0.176)$ & $0.218(0.420)$ \\
Mean & $0.096(0.548)$ & $1.275(0.683)$ & $0.233(0.456)$ \\
\hline
\end{tabular}

Note: $S S D=$ Sum of squared deviations; $r=$ Harpending's Raggedness index. $P$ values are displayed in parentheses, where the statistical analysis reached significant level at $p<0.05$. Names of population, corresponding to these abbreviations is given in Table 1.

\section{Median joining network of haplotypes and phylogenetic analysis}

A neighbor-joining tree was constructed based on 34 haplotypes $(\mathrm{H} 1-\mathrm{H} 34)$ in 147 chickens from the 14 different breeds (Table 1), meanwhile the Meleagris gallopavo (AY398680) was used as an outgroup (Figure 2). The reliability of $\mathrm{NJ}$ tree topology was calculated with 1000 bootstrap replications. As shown in Figure 3, four clusters (A, B, C and D) were found. Eighteen haplotypes ( $\mathrm{H} 1$ - H14, H17, H18, H24, H34) were classified as cluster $\mathrm{A}, \mathrm{H} 1$ was the dominant haplotype, present in $44.64 \%$ of all individuals in cluster A $(25 / 56=44.64 \%)$; eight haplotypes (H15, H16, H29,H28, H30, H31, H32 and H33) were classified as cluster $\mathrm{B}, \mathrm{H} 32$ was the dominant haplotype, present in $45.71 \%$ of all individuals in cluster B $(32 / 70=45.71 \%)$; five haplotypes $(H 19$, $\mathrm{H} 20, \mathrm{H} 21 \mathrm{H} 22$ and H23) were classified as cluster $\mathrm{C}$, $\mathrm{H} 23$ was the dominant haplotype, present in $64.29 \%$ of all individuals in cluster C $(9 / 14=64.29 \%) ; \mathrm{H} 25$, $\mathrm{H} 26$ and $\mathrm{H} 27$ these three haplotypes were classified as cluster D.

The result of Median-joining network (Figure 3) based on the 34 haplotypes constructed by the NETWORK program showed that the haplotypes were also divided into four clades. The results showed the similarity to the NJ tree. From Figure 3, we can find that $\mathrm{H} 1, \mathrm{H} 23$ and $\mathrm{H} 32$ were mainly distributed in the center of the median joining network, with derivative haplotypes spreading outwards from it. Therefore, we think that $\mathrm{H} 1, \mathrm{H} 23$ and $\mathrm{H} 32$ are the earliest haplotypes.

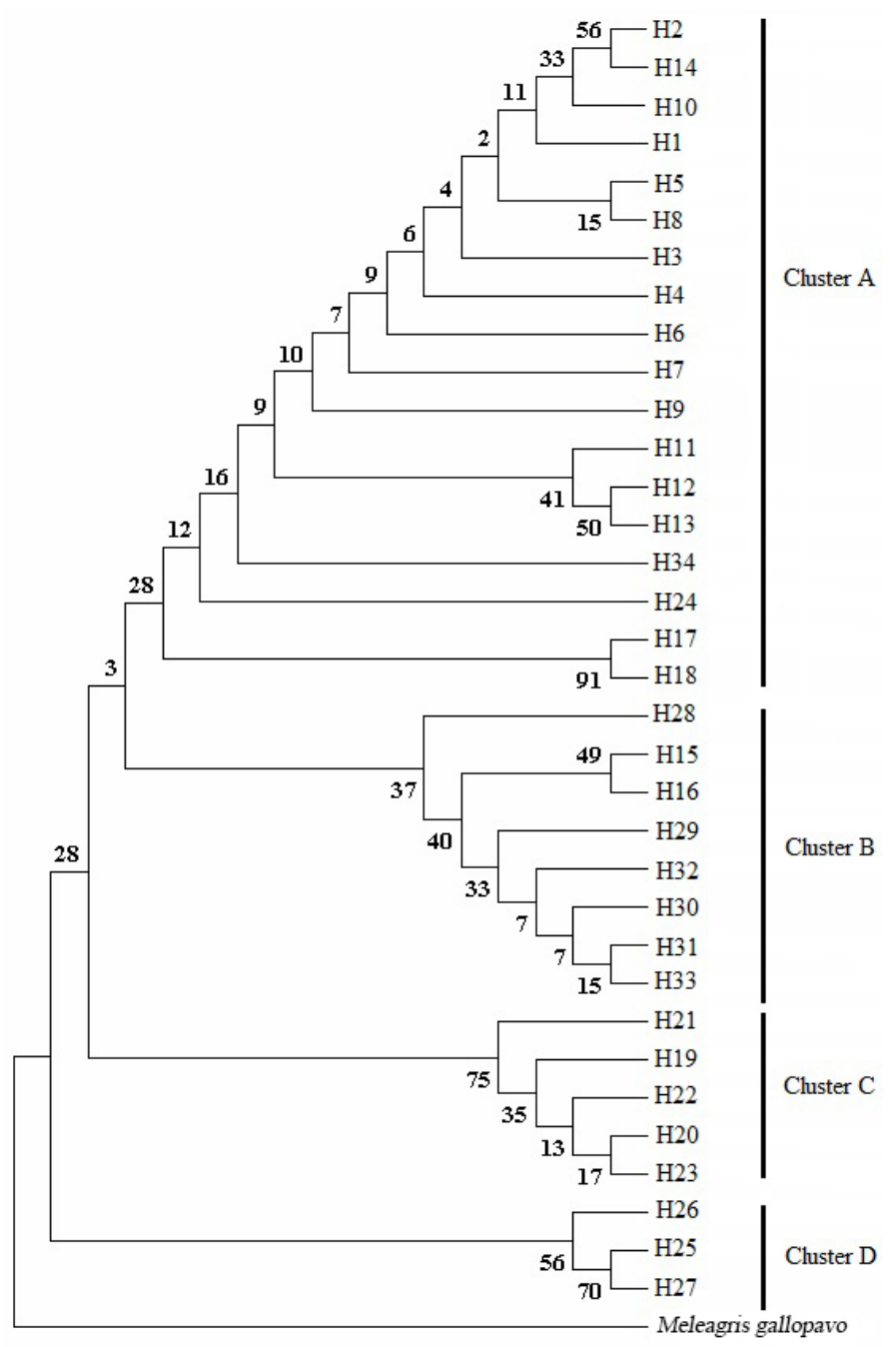

Figure 2 - The Neighbour-joining tree based on 34 D-loop haplotypes of fourteen chicken breeds. The tree was constructed on the basis of Kimura two-parameter distance. The numbers at the major nodes represent the percentage bootstrap values for interior branches after 1000 replications. 


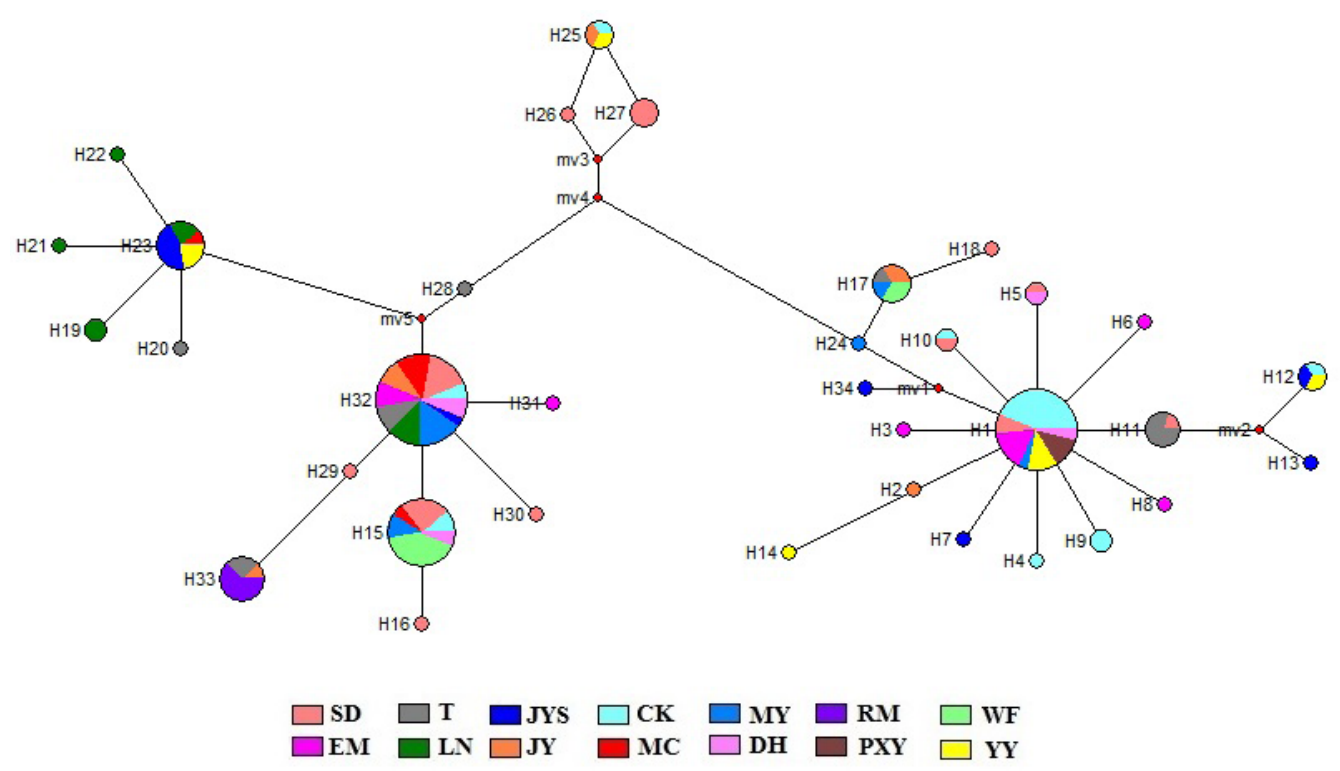

Figure 3 - Median-Joining network profile of the mtDNA D-loop haplotypes observed in 14 chicken breeds. Each circle represents an haplotype. The circle size is proportional to its total frequency and different colored circles represent different chicken breeds. $\mathrm{H} 1=$ haplotype $1, \mathrm{H} 2=$ haplotype $2, \ldots . . \mathrm{H} 34=$ haplotype 34 .

\section{DISCUSSION}

In this study, we analyzed partial mtDNA D-loop sequences from 147 chickens sampled in Sichuan, province of China. The results revealed that these chicken breeds had a rich genetic diversity. It is generally known that haplotype diversity and nucleotide diversity of mtDNA are the important index for assessing population polymorphism and genetic differentiation (Liu et al., 2006b; Liang et al., 2016). The haplotype diversity results showed that the genetic variation of $S D, J Y, E M, L N, J Y S, Y Y$ and $D H$ in this study were abundant with the haplotype diversity value more than 0.80. However, the PXY, WF and RM breeds showed lower genetic diversity levels, the haplotype diversity value are lower than 0.55 . Observing this result, we think that long-term artificial selection may lead to reduced nucleotide diversity in these commercial chicken breeds. Although the $\mathrm{DH}$ is a commercial chicken breed, it was bred by Sichuan Dahen Poultry Breeding Co., Ltd, located at Hanchang Town, Chengdu City of Sichuan Province, using Chinese indigenous breeds, and only had a short-term artificial selection (Zhou et al., 2017). The PXY maybe suffered a longterm or high-strength artificial selection in the genetic resources conservation procedure. This result showed similar status to a previous study of eight Sichuan indigenous chicken breeds based on microsatellite analysis. Meanwhile, the haplotype and nucleotide diversity from CK, LN, JY, EM and MC population were higher than the results of Liu et al., (2017). These results were caused by different researched regions of mtDNA sequence and methods.

In the current study, most of the variation (80.80\%) was found within-population in terms of the AMOVA analysis results. Thus, this result indicated that there was a significant differentiation among the fourteen breeds. Recently, some researchers found that Fst value is an important indicator of genetic differentiation between breeds (Liang et al., 2016; Al-Araimi et al., 2017). So, the result of the coefficient of genetic differentiation and gene flow between breeds in our study suggests that some chicken breeds have undergone genetic differentiation at places of equal regions. For instance, the SD from Xingwen county and PXY from Pengzhou county have a close population genetic relationship with the $T$ chicken and $J Y$ chicken, respectively ( $F s t=0.006, \mathrm{Nm}=71.666$; $\mathrm{Fst}=0.006, \mathrm{Nm}=82.404$ ). This indicated that the SD and PXY was introduced from the remote region of Sichuan. Moreover, YY and JYS chickens from Liangshan prefecture regions were the most similar ( $\mathrm{Da}=0.0001, \mathrm{Dxy}=0.0203)$ suggesting that the genetic distance of the two breeds is very close, and maybe more frequent gene exchange occured ( $F s t=0.009, N m=56.91$ ).

Some morphology traits of indigenous chickens are similar, so it is difficult to study the taxonomy and phylogeny according to the different morphology (Nico e et al., 2011; Cai et al., 2013). Molecular phylogenetic and analytical techniques were the important methods to identify genetic divergence of chicken. In this study, fourteen chicken breeds were categorized into four 
divergent clusters referred as cluster A, B, C and D (Figure 3). Cluster $A$ is the most representative when considering the number of haplotypes among the Sichuan indigenous chicken breeds, which is consistent with the findings of previous studies (Liu et al., 2006; Zhang et al., 2017) reporting that most domesticated chicken carry this cluster. It could be speculated that cluster A was the most universal phylogenic cluster in the history of chicken evolution. Meanwhile, cluster $C$ was mainly shared by three breeds (LN, JYS and YY), which should be an important native lineage in Liangshan autonomous prefecture in Sichuan.

\section{CONCLUSION}

We sequenced and compared 11 indigenous chicken breeds of Sichuan province and 3 commercial chicken breeds in this study. The results indicated that genetic diversity of $\mathrm{DH}$ population was the highest and that the population of PXY was the lowest. Additionally, hybridization may exist within or among different breeds. To sustainably utilize the germplasm resources of these indigenous chickens and reveal the clear gene flow among different population, further strategies should be developed to protect the current chicken breeds and more genetics or genomics studies need to be conducted.

\section{CONFLICTS OF INTEREST}

The authors declare no conflicts of interest regarding the publication of this paper.

\section{ACKNOWLEDGMENTS}

We are grateful to the editors and reviewers for the careful reviews and the honest advice. Particular thanks go to all of the people who helped with sample collection. This work was funded by the Project of National Science and Technology Plan in China (2015BAD03B03), the National modern agricultural technology system construction of China (CARS-41-G04), Key Technology Support Program of Sichuan Province (2016NYZ0043, 2016NZ0104, 2018NZDZX0004).

\section{REFERENCES}

Al-Araimi NA, Al-Atiyat RM, Gaafar OM, Vasconcelos R, Luzuriaga-Neira $A$, Eisa MO, et al. Maternal genetic diversity and phytogeography of native Arabian goats. Livestock Science 2017;206: 88-94.

Bandelt HJ, Forster P, Rohl A. Median-joining networks for inferring intraspecific phylogenies. Molecular Biology and Evolution 1999;16:3748.
Brown MW, Prager EM, Wang A, Wilson AC. Mitochondrial DNA sequences of primates:Tempo and mode of evolution. Journal of Molecular Evolution 1982;18:225-239.

Cai X, Yu S, Mipam T, Zhang X, Yue B. Phylogenetic lineages of Monopterus albus (Synbranchiformes: Synbranchidae) in China inferred from mitochondrial control region. Journal of Zoological Systematics and Evolutionary Research 2013;51:38-44.

Czerneková V, Kott T, Majzlík I. Mitochondrial D-loop sequence variation among Hucul horse. Czech Journal of Animal Science 2013;58:437442.

Ceccobelli S, Di Lorenzo P, Lancioni H, Monteagudo Ibáñez LV, Tejedor MT, Castellini C, et al. Genetic diversity and phylogeographic structure of sixteen Mediterranean chicken breeds assessed with microsatellites and mitochondrial DNA. Livestock Science 2015;175:27-36.

Di Lorenzo P, Ceccobelli S, Panella F, Attard G, Lasagna E. The role of mitochondrial DNA to determine the origin of domestic chicken. World's Poultry Science Journal 2015;71:311-318.

Eltanany MA, Hemeda SA. Deeper insight into maternal genetic assessments and demographic history for Egyptian indigenous chicken populations using mtDNA analysis. Journal of Advanced Research 2016;7:615-623.

Excoffier L, Laval G, Schneider S. Arlequin (version 3.0): an integrated software package for population genetics data analysis. Evolutionary Bioinformatics Online 2005:1:47-50.

Fu Y, Niu D, Luo J, Ruan H, He GQ, Zhang YP. Studies of the origin of Chinese domestic fowls. Acta Genetica Sinica 2001;28:411-417.

Felsenstein J. Confidence limits on phylogenies: an approach using the bootstrap. Evolution 1985;39:783-791.

Groeneveld LF, Lenstra JA, Eding H, Toro MA, Scherf B, Pilling D, et al. Genetic diversity in farm animals-a review. Animal Genetics 2010;41:631

Ivanković A, Paprika S, Ramljak J, Dovć P, Konjaćić M. Mitochondrial DNAbased genetic evaluation of autochthonous cattle breeds in Croatia. Czech Journal of Animal Science 2014;59:519-528.

Izadi F, Ritland C, Cheng KM. Genetic diversity of the major histocompatibility complex region in commercial and noncommercial chicken flocks using the LEI0258 microsatellite marker. Poultry Science 2011;90:2711-2717.

Kumar S, Stecher G, Tamura K. MEGA 7: Molecular Evolutionary Genetics Analysis version 7.0 for bigger datasets. Molecular Biology and Evolution 2016:3:1870-1874

Liang H, Guo S, Li Z, Luo X, Zou G. Assessment of genetic diversity and population structure of swamp eel Monopterus albus in China. Biochemical Systematics and Ecology 2016;68:81-87.

Librado P, Rozas J. DnaSP v5: a software for comprehensive analysis of DNA polymorphism data. Bioinformatics 2009;25:1451-1452.

Li HF, Zhu WQ, Song WT, Shu JT, Han W, Chen KW. Origin and genetic diversity of Chinese domestic ducks. Molecular Phylogenetics and Evolution 2010;57:634-640.

Liu X, Zhang P, Zhang G, Li S, Zhang L, Xu Z, Ma T, Li D. A comparison of genetic diversity of COX-III gene in lowland chickens and Tibetan chickens. BioMed Research International 2017;ID8064613:1-13.

Liu YP, Wu GS, Yao YG, Miao YW, Luikart G, Baig M, et al. Multiple maternal origins of chickens: out of the Aisan jungles. Molecular Phylogenetics and Evolution 2006a;38:12-19.

Liu RY, Yang GS, Lei CZ. The genetic diversity of mtDNA D-loop and the origin of Chinese goats. Acta Genetic Sinica 2006b;23:420-428. 
Yu C, Qiu M, Jiang X, Zhang Z, Du H, Li

$Q$, Xia B, Song $X$, Hu C, Xiong $X$, Yang $L$, Peng $\mathrm{H}$, Chen J, Wang $\mathrm{Y}$, Yang $\mathrm{C}$

Genetic Diversity and Phyletic Evolution of Eleven Chinese Indigenous and Three Commercial Chicken Breeds by mtDNA Sequences

Moiseyeva IG, Romanov MN, Nikiforov AA, Sevastyanova AA, Semyenova SK. Evolutionary relationships of Red Jungle Fowl and chicken breeds. Genetics Selection Evolution 2006;35:403-423.

Muchadeyi FC, Eding H, Simianer H, Wollny CBA, Groeneveld E, Weigend S. Mitochondrial DNA D-loop sequences suggest a Southeast Asian and Indian origin of Zimbabwe an village chickens. Animal Genetics 2008:39:615-622.

Mwacharo JM, Bjørbstad G, Mobegi V, Nomura K, Hanada H, Amano T, et al. Mitochondrial DNA reveals multiple introductions of domestic chicken in East Africa. Molecular Phylogenetics and Evolution 2011;58:374-382.

Nico LG, Sharp P, Collins TM. Imported Asian swamp eels (Synbranchidae: Monopterus) in north american live food markets: potential vectors of non-native parasites. Aquatic Invasions 2011;6:69-76.

Okumu ON, Ngeranwa JJN, Binepal YS, Kahi AK, Bramwel WW, Ateya LO, et al. Genetic diversity of indigenous chickens from selected areas in Kenya using microsatellite markers. Journal of Genetic Engineering and Biotechnology 2017;15:489-495.
Walker SM, Prodohl PA, Fletcher HL, Hanna RE, Kantzoura V, Hoey EM, et al. Evidence for multiple mitochondrial lineages of Fasciola hepatica (liver fluke) within infra populations from cattle and sheep. Parasitology Research 2007;101:117-125.

Wu YP, Huo JH, Xie JF, Liu LX, Wei QP, Xie MG, et al. Phylogeography and origin of Chinese domestic chicken. Mitochondrial DNA 2014:25(2):126-130.

Zhang L, Zhang P, Li Q, Gaur U, Liu Y, Zhu Q, et al. Genetic evidence from mitochondrial DNA corroborates the origin of Tibetan chickens. PLoS One 2017;12(2):e0172945.

Zhou YG, Xiong Y, Yang CW, Jiang XS, Ran JS, Jin J, et al. Experimental verification of CAPN1 and CAST gene polymorphisms in different generations of Da-Heng broilers. BioMed Research International 2017;ID7968450. 